\title{
A transcriptome-base analisys of lilac apical complexes in vivo and in vitro
}

\author{
Krinitsina A.A. ${ }^{1 *}$, Speranskaya A.S. ${ }^{1}$, Churikova O.A. ${ }^{1}$, Logacheva M.D. ${ }^{1}$, \\ Konorov E.A. ${ }^{2}$, Belenikin M.S. ${ }^{3}$ \\ ${ }^{1}$ Lomonosov Moscow State University, Moscow, Russia \\ 2 Vavilov Institute of General Genetics, RAS, Moscow, Russia \\ ${ }^{3}$ Moscow Institute of Physics and Technology, Dolgoprudny, Russia \\ *e-mail: ankrina@gmail.com
}

Comparative transcriptome analysis of vegetative apices of Syringa vulgaris L. during physiological rest or in phase of active growth in vivo and in vitro were carried out. For transcriptome analysis the two type of lilac's shoots were used: (1) the aseptic culture of Syringa vulgaris L. shoots cultivated in the lab since 2008, (2) the adult plants growing in the open ground of MSU Botanical Garden. For experiment the aseptic microshoots were precultivated under slow-growth culture condition during 270 days after then apexes of one half of shoots were cutted and fixed in RNA-later. The remaining shoots were transferred to normal condition and growing for 28 days. Likewise the vegetative apices of adult $S$. vulgaris shrubs were collected under physiological rest (in winter) and from active growth (in springtime). The total RNA was purified with RNeasy Plant Mini Kit (Qiagen) from $20 \mathrm{mg}$ of each fixed samples. The libraries were prepared using NEBNext ${ }^{\circledR}$ Poly(A) mRNA Magnetic Isolation Module and NEBNext ${ }^{\circledR}$ Ultra RNA Library Prep Kit for Illumina (NEB) and sequenced using HiSeq 2500 System. Reference transcriptome was assembled by Trinity, transcript quantification were made by Salmon and differential expression analysis were conducted using edgeR. A pairwise comparison of samples showed different level transcripts of two $\beta$-glucosidase isoforms, five superoxide dismutase isoforms, four peroxidase isoforms and three xyloglucan xylo glucosyltransferase isoforms in slow-growth condition in vitro vs. physiological rest lilac's plant apices in vivo. And different level transcripts of seven superoxide dismutase isoforms, three $\beta$-glucosidase isoforms and cytochrome p450 isoforms in lilac's shoot apices of active growth plants in vivo vs. in vitro. 